\title{
Raphaëlle Branche, Nadine Picaudou, Pierre Vermeren (dir.), Autour des morts de guerre. Maghreb - Moyen-Orient
}

Paris, Publications de la Sorbonne, coll. «Internationale », 2013, 236 p.

\section{Cécile Boëx}

\section{OpenEdition \\ Journals}

Édition électronique

URL : http://journals.openedition.org/assr/28193

DOI : $10.4000 /$ assr.28193

ISSN : $1777-5825$

Éditeur

Éditions de l'EHESS

Édition imprimée

Date de publication : 31 décembre 2016

Pagination : 280

ISSN : 0335-5985

Référence électronique

Cécile Boëx, « Raphaëlle Branche, Nadine Picaudou, Pierre Vermeren (dir.), Autour des morts de guerre. Maghreb - Moyen-Orient », Archives de sciences sociales des religions [En ligne], 176 | octobredécembre 2016, mis en ligne le 17 juillet 2017, consulté le 24 septembre 2020. URL : http:// journals.openedition.org/assr/28193; DOI : https://doi.org/10.4000/assr.28193

Ce document a été généré automatiquement le 24 septembre 2020.

(c) Archives de sciences sociales des religions 


\section{Raphaëlle Branche, Nadine Picaudou, Pierre Vermeren (dir.), Autour des morts de guerre. Maghreb - Moyen-Orient}

Paris, Publications de la Sorbonne, coll. «Internationale », 2013, 236 p.

\section{Cécile Boëx}

\section{RÉFÉRENCE}

Raphaëlle Branche, Nadine Picaudou, Pierre Vermeren (dir.), Autour des morts de guerre. Maghreb - Moyen-Orient, Paris, Publications de la Sorbonne, coll.

«Internationale », 2013, $236 \mathrm{p}$.

1 Interroger les guerres au Proche et au Moyen-Orient autrement que par le prisme de l'actualité et de la géopolitique constitue une entreprise louable et ardue. C'est ce que proposent Raphaëlle Branche, Nadine Picaudou et Pierre Vermeren dans cet ouvrage collectif qui réunit des spécialistes de la région issus de diverses disciplines pour penser la violence, la mort et la mémoire autour d'un objet commun : « les morts de guerre ». D'emblée, ce choix terminologique marque l'ambition d'ouvrir une nouvelle voie de questionnement au sein d'une littérature particulièrement prolixe sur le martyr. Il s'agit ici d'élargir la réflexion en l'inscrivant dans une perspective comparative située à la jonction de l'histoire, de l'anthropologie et des sciences politiques. L'objectif de l'ouvrage est ainsi de saisir l'expérience de la guerre pour éclairer certains des enjeux sociétaux qui se nouent dans les processus de mise en récit de la mort et dans la commémoration des défunts, qu'il s'agisse de combattants, de civils ou de figures politiques.

2 Les contributions mettent en résonnance divers contextes guerriers (guerres d'indépendance, guerres civiles, guerres nationales) et croisent deux perspectives 
analytiques principales. La première concerne le culte des morts de guerre et épouse largement les contours des réflexions antérieures sur le martyre. Néanmoins, il s'agit ici de retracer les processus sémantiques et iconographiques, au croisement du politique et du religieux, qui permettent de transformer les morts de guerre en martyrs. Ce changement de statut des défunts ne va pas de soi et met au jour «des espaces de confrontations symboliques entre les acteurs familiaux, religieux et politiques » («Introduction», p.12). La question du culte des morts est donc appréhendée du point de vue des acteurs et des pratiques, mais aussi et surtout, de celui des politiques publiques de prise en charge de la mort et de la commémoration. C'est le deuxième axe qui traverse et domine l'ouvrage, s'inscrivant dans une histoire politique du deuil et de la commémoration " par le haut » (États, partis, milices).

3 Emmanuel Alcaraz examine l'instrumentalisation politique de la mémoire de la guerre d'indépendance algérienne en analysant la sémantique du monument aux martyrs d'Alger ainsi que celle du Musée national du moudjahid. L'auteur montre comment, lors des commémorations qui ont lieu dans le premier, les dirigeants revendiquent l'héritage des martyrs en mobilisant les registres du nationalisme algérien et de la foi musulmane. De même, à partir d'une description détaillée de la scénographie du Musée, il éclaire certaines modalités de la gestion autoritaire de la mémoire de cette guerre. Le Liban Sud, région fortement marquée par la présence de la communauté chiite, offre une perspective différente sur la gestion de la mort dans le contexte d'un État défaillant. Kinda Chaïb interroge plus particulièrement les conséquences d'une guerre qui dure depuis plus de soixante ans avec Israël sur la ritualité funéraire. Au moyen d'une analyse des représentations et de la visibilité des martyrs dans l'espace public (portraits, cimetières), l'auteur suggère que la présence massive et quotidienne des morts de guerre a engendré la diffusion de nouveaux codes et de nouvelles pratiques funéraires, lesquels ont eu pour effet de rendre la catégorie "martyr » extensible, notamment dans le cas de la mort de jeunes hommes non combattants.

Sümbül Kaya et Étienne Copeaux traitent d'un tout autre cas de figure et montrent comment, depuis 1984, l'État turc consolide son pouvoir sécuritaire à travers le « langage des obsèques » des soldats tués dans le Sud-Est. Immuable dans son approche de la question kurde, l'État impose son interprétation des faits et exige un consensus de la part des citoyens au moyen de discours et de mises en scène rigoureusement orchestrées qui brouillent les frontières entre le passé et le présent, mais aussi entre le politique et le religieux. La prise en charge totale (matérielle, financière et rituelle) de la mort des soldats engendre un processus de réappropriation politique adossé à une communication médiatique de masse. Ce verrouillage sémantique, basé sur l'exaltation du sentiment d'appartenance nationale, entrave toute forme de débat. De ce point de vue, les auteurs font remarquer que l'évolution des moyens de communication et l'avènement des réseaux sociaux n'ont pas forcément altéré les représentations de la mort des soldats.

5 Jean-Pierre Peyroulou rend compte au contraire de la plasticité de la figure du martyr en Algérie, qui évolue au gré d'enjeux politiques et mémoriels. Il observe ainsi des usages fluctuants de la commémoration des « martyrs » des massacres du Constantinois de mai 1945. Élément central du martyrologe algérien jusqu'en 1954, il est pourtant éclipsé de l'histoire et de la mémoire algérienne pendant la guerre d'indépendance et durant les trois premières décennies de la construction de l'État. Cette rupture mémorielle avec les morts du 8 mai 1945 s'inscrit dans la volonté du FLN d'évincer les 
courants nationalistes concurrents. Dès lors, c'est la figure du moudjahid qui est exaltée. Néanmoins pendant les années 1990, la guerre civile ramène les martyrs du colonialisme sur le devant de la scène alors que l'extrême violence des groupes armés et de l'État réinterrogent les fondements de la nation. Ce sont d'autres usages politiques de la mémoire de guerre qui nous sont présentés ensuite. "Oubliés de l'histoire ", la mémoire controversée des anciens combattants marocains et tunisiens de l'armée française est toujours soumise aux conjonctures historiques et aux relations des pays d'origine avec la France. À partir de témoignages, Christophe Giudice examine comment ces hommes se sont réinsérés dans leurs sociétés durant la période coloniale puis après les indépendances. L'auteur montre également comment les États se sont réapproprié la symbolique commémorative de l'armée française pour glorifier les martyrs et les héros de l'indépendance, aux dépens des soldats de l'armée d'Afrique.

Les contributions de Stéphane Malsagne et de Dima de Clerck nous emmènent ensuite sur le terrain de la guerre civile au Liban, qui met au jour des usages spécifiques de la gestion des morts. La première s'appuie sur l'analyse des obsèques de sept leaders politiques et religieux aux origines confessionnelles diverses, assassinés pendant la guerre civile (1975-1990). Il s'agit de montrer comment le deuil articule sphères privées et publiques dans l'organisation des funérailles, puisant à la fois dans des traditions, mais créant aussi des rituels propres à la guerre. Cette catégorie de funérailles déploie ainsi une grammaire politique complexe qui mobilise des jeux d'alliances inter et intracommunautaires, ainsi que des stratégies de régulation de la violence. Dima de Clerck s'intéresse quant à elle à la place des morts chez les chrétiens du sud du MontLiban, région marquée par des affrontements violents avec la communauté druze durant la guerre civile. Qu'en est-il de la place physique et symbolique des morts lorsque les vivants se sont vus contraints de quitter leur village d'origine? C'est la question qui sert de point de départ à une analyse qui croise une double problématique : celle des morts de guerre chrétiens comme enjeu de réconciliation (et de retour) et celle du « remodelage » des pratiques funéraires et commémoratives des chrétiens depuis 1983. Ces deux éléments permettent d'éclairer les rapports antagonistes à la terre et aux origines qui divisent la communauté chrétienne après les réconciliations. Alors que les familles les plus éprouvées souhaitent pour la plupart rompre avec le village, d'autres s'emploient au contraire à renouer avec le lieu originel, soit par nostalgie, soit par la volonté d'affirmer une identité qui nourrit une posture militante.

$7 \quad$ Les deux dernières contributions interrogent les codes visuels et performatifs mobilisés dans la représentation et la narration de la guerre et de ses morts en contexte chiite. Ici, le martyre de Husayn constitue un répertoire symbolique dominant, même si l'industrie cinématographique en Iran a introduit des formes de mises en récit alternatives, qui n'en restent pas moins extrêmement codifiées. Agnès Devictor examine les films de fiction réalisés pendant la guerre Iran-Irak (1980-1988) qui, à rebours des conventions du cinéma de guerre, représentent la mort de soldats iraniens dans la temporalité même du conflit. Par quels dispositifs de mise en scène ces morts deviennent-elles acceptables? Ceux-ci sont variés et puisent, selon les catégories de films, dans les registres du religieux ou du cinéma hollywoodien. L'auteur montre donc ici que la variable culturaliste "martyrophile » ne suffit pas à expliquer la diversité des formes narratives et esthétiques. Néanmoins, le régime de la fiction reste une condition incontournable pour représenter ces morts. Pour sa part, Michel Tabet s'intéresse aux aspects visuels des mises en scène du martyre fondateur de Husayn et des manières 
dont celles-ci évoluent à la lumière des nouvelles technologies de communication. Il propose donc d'appréhender des formes de "flexibilités rituelles » à partir de l'analyse des célébrations de 'Achoura de Nabatieh, au Liban Sud. Les variations de l'image du martyre de Husayn sont à la fois saisies dans les expressions sociales et politiques du rituel tout comme dans ses transcriptions visuelles et médiatiques. Aussi, les usages symboliques différenciés vont bien au-delà des usages cérémoniels des images du martyre pour nourrir une culture guerrière, comme dans le cas du Hezbollah ou pour faire du rituel un événement médiatique ou un spectacle à part entière.

Une conclusion générale aurait sans doute permis d'éclaircir les contours de l'objet " mort de guerre », tout comme ses apports heuristiques dans la compréhension du fait guerrier et de son impact sur les sociétés du Maghreb et du Moyen-Orient. De ce point de vue, une réflexion plus globale sur les enjeux politiques, institutionnels et religieux qui traversent cet objet aurait pu consolider l'ossature problématique de l'ouvrage. En effet, l'éclectisme thématique et analytique des contributions est parfois déroutant. Mais l'angle d'approche proposé ici n'en reste pas moins stimulant, d'autant plus qu'il regroupe des travaux originaux de jeunes chercheurs ancrés dans des terrains de longue durée. La diversité des contextes et des questionnements qu'ils appréhendent jettent les bases d'un champ de réflexion qui ne peut effectivement pas faire l'économie de la pluridisciplinarité et du comparatisme. Par ailleurs, comme certaines contributions le suggèrent, le développement des technologies de communication préfigure de nouvelles modalités de représentation et de remémoration des morts de guerre; un phénomène d'autant plus prégnant depuis les bouleversements politiques qui traversent la région depuis 2011. Cet ouvrage est donc indispensable pour qui cherche à mieux comprendre ce que les guerres font aux sociétés, et à saisir, dans leur complexité, les bouleversements d'une partie du monde particulièrement traversée par des formes de violences politiques et guerrières multiples. 